\title{
Characteristic Properties of Type-2 Smarandache Ruled Surfaces According to the Type-2 Bishop Frame in $E^{3}$
}

\author{
Ibrahim Al-Dayel $\mathbb{D}^{1}$ and E. M. Solouma ${ }^{1,2}$ \\ ${ }^{1}$ Department of Mathematics and Statistics, College of Science, Imam Mohammad Ibn Saud Islamic University, Saudi Arabia \\ ${ }^{2}$ Department of Mathematics and Information Science, Faculty of Science, Beni-Suef University, Egypt
}

Correspondence should be addressed to Ibrahim Al-Dayel; iaaldayel@imamu.edu.sa

Received 9 July 2021; Revised 17 August 2021; Accepted 11 September 2021; Published 22 October 2021

Academic Editor: Shyamal Kumar Hui

Copyright ( 2021 Ibrahim Al-Dayel and E. M. Solouma. This is an open access article distributed under the Creative Commons Attribution License, which permits unrestricted use, distribution, and reproduction in any medium, provided the original work is properly cited.

In this paper, we define and investigate a special kind of ruled surfaces called type-2 Smarandache ruled surfaces related to the type-2 Bishop frame in $\mathbf{E}^{3}$. From this point and depending on the type-2 Bishop curvature, we provide the necessary and sufficient conditions that allow these surfaces to be developable in a minimal amount of time. Furthermore, an example is given to clear the results.

\section{Introduction}

In the classical differential geometry, the theory of ruled surfaces is one of its branches which has been developed by several researchers. A ruled surface is generally defined as the set of a family of straight lines that depend on a parameter that is mentioned as the ruled surface's rulings. A ruled surface's parametric representation is $Y(\sigma, v)=c(\sigma)+v X(\sigma)$ where $c(\sigma)$ is the base curve of $Y(\sigma, v)$ and $X(\sigma)$ define the ruling directions [1, 2]. Surfaces' developability and minimalist notions are two of their most important properties. One of the most interesting points is the study of ruled surfaces with different moving frames, as seen in this example [3-7].

The Smarandache curve in Euclidean and Minkowski spaces is the curve whose position vector is made by Frenet frame vectors on another regular curve [8-11]. Several researchers [12-20] have recently studied Smarandache curves in Minkowski and the Euclidean spaces.

In this work, in $\mathbf{E}^{3}$, we introduce the definitions of type- 2 Smarandache ruled surfaces using the type-2 Bishop frame, namely, $\mu_{1} \mu_{2}, \mu_{1} B$, and $\mu_{2} B$ type- 2 Smarandache ruled surfaces. Our main results are presented in theorems that look into the necessary and sufficient conditions for those surfaces to be developable and minimal. Throughout the response, an example with illustrations is created.

\section{Preliminaries}

Let $\mathbf{E}^{3}$ be a 3-dimensional Euclidean space provided with the metric

$$
\langle,\rangle=d u_{1}^{2}+d u_{2}^{2}+d u_{3}^{2},
$$

where $\left(u_{1}, u_{2}, u_{3}\right)$ is the rectangular coordinate system of $\mathbf{E}^{3}$.

Representing the moving Frenet frame along its regular curve $\psi$ by $\{T, N, B\}$ in conjunction with curvature functions $\kappa$ and $\tau$ in $\mathbf{E}^{3}$, the Frenet formula is given as follows [1]:

$$
\frac{d}{d \sigma}\left(\begin{array}{c}
T(\sigma) \\
N(\sigma) \\
B(\sigma)
\end{array}\right)=\left(\begin{array}{ccc}
0 & \kappa(\sigma) & 0 \\
-\kappa(\sigma) & 0 & \tau(\sigma) \\
0 & -\tau(\sigma) & 0
\end{array}\right)\left(\begin{array}{c}
T(\sigma) \\
N(\sigma) \\
B(\sigma)
\end{array}\right),
$$

where $\langle T, T\rangle=\langle N, N\rangle=\langle B, B\rangle=1$ and $\langle T, N\rangle=\langle T, B\rangle$ $=\langle N, B\rangle=0$. 
For any arbitrary curve $\psi$ with $\tau \neq 0$ in $\mathbf{E}^{3}$, the type-2 Bishop frame of $\psi$ is given as follows [21]:

$$
\frac{d}{d \sigma}\left(\begin{array}{c}
\mu_{1}(\sigma) \\
\mu_{2}(\sigma) \\
B(\sigma)
\end{array}\right)=\left(\begin{array}{ccc}
0 & 0 & -k_{1}(\sigma) \\
0 & 0 & -k_{2}(\sigma) \\
k_{1}(\sigma) & k_{2}(\sigma) & 0
\end{array}\right)\left(\begin{array}{c}
\mu_{1}(\sigma) \\
\mu_{2}(\sigma) \\
B(\sigma)
\end{array}\right)
$$

where $k_{1}$ and $k_{2}$ are the type-2 Bishop curvatures and satisfying

$$
\left(\begin{array}{c}
T(\sigma) \\
N(\sigma) \\
B(\sigma)
\end{array}\right)=\left(\begin{array}{ccc}
\sin \theta(\sigma) & -\cos \theta(\sigma) & 0 \\
\cos \theta(\sigma) & \sin \theta(\sigma) & 0 \\
0 & 0 & 1
\end{array}\right)\left(\begin{array}{c}
\mu_{1}(\sigma) \\
\mu_{2}(\sigma) \\
B(\sigma)
\end{array}\right),
$$

where $\theta(\sigma)=\arctan \left(k_{2} / k_{1}\right)$ and

$$
\begin{aligned}
& k_{1}=-\tau \cos \theta(\sigma), \\
& k_{2}=-\tau \sin \theta(\sigma) .
\end{aligned}
$$

Definition 1. [21]. $\mu_{1} \mu_{2}$ type-2 Smarandache curves of the curve $\psi(\sigma)$ via $\left\{\mu_{1}, \mu_{2}, B\right\}$ are given as

$$
\beta\left(\sigma^{*}(\sigma)\right)=\frac{1}{\sqrt{2}}\left(\mu_{1}(\sigma)+\mu_{2}(\sigma)\right) .
$$

Definition 2. [21]. $\mu_{1} B$ type-2 Smarandache curves of the curve $\psi(\sigma)$ via $\left\{\mu_{1}, \mu_{2}, B\right\}$ are given as

$$
\gamma\left(\sigma^{*}(\sigma)\right)=\frac{1}{\sqrt{2}}\left(\mu_{1}(\sigma)+B(\sigma)\right)
$$

Definition 3. [21]. $\mu_{2} B$ type-2 Smarandache curves of the curve $\psi(\sigma)$ via $\left\{\mu_{1}, \mu_{2}, B\right\}$ are given as

$$
\delta\left(\sigma^{*}(\sigma)\right)=\frac{1}{\sqrt{2}}\left(\mu_{2}(\sigma)+B(\sigma)\right) .
$$

A ruled surface $v$ in $\mathbf{E}^{3}$ can be reparametrized as

$$
v(\sigma, v)=\psi(\sigma)+v \chi(\sigma)
$$

where $\psi(\sigma)$ is really the base curve and $\chi(\sigma)$ is its unit which defines a space curve that characterizes the straight line's direction [22].

$v$ 's unit normal vector $N$ is given as follows [23]:

$$
\mathbf{U}=\frac{v_{\sigma} \times v_{v}}{\left\|v_{\sigma} \times v_{v}\right\|}
$$

where $v_{\sigma}=\partial v / \partial \sigma$ and $v_{v}=\partial v / \partial v$. The Gaussian curvature $K$ and the mean curvature $H$ are given as follows [23]:

$$
\begin{aligned}
K & =\frac{\ell n-m^{2}}{E G-F^{2}}, \\
H & =\frac{E n+G \ell-2 m F}{2\left(E G-F^{2}\right)},
\end{aligned}
$$

where $E=\left\|Y_{\sigma}\right\|^{2}, F=\left\langle Y_{\sigma}, Y_{v}\right\rangle, G=\left\|Y_{v}\right\|^{2}, \ell=\left\langle Y_{\sigma \sigma}, \mathbf{U}\right\rangle, m$ $=\left\langle Y_{\sigma v}, \mathbf{U}\right\rangle$, and $n=\left\langle Y_{v v}, \mathbf{U}\right\rangle$. The normal curvature, geodesic curvature, and geodesic torsion that connects the curve $\psi(\sigma)$ on $Y$ are computed as follows:

$$
\kappa_{n}=\left\langle\psi^{\prime \prime}, \mathbf{U}\right\rangle, \kappa_{g}=\left\langle\mathbf{U} \times T, T^{\prime}\right\rangle, \tau_{g}=\left\langle\mathbf{U} \times \mathbf{U}^{\prime}, T^{\prime}\right\rangle .
$$

Definition 4. A ruled surface is developable if and only if $K$ $=0$ and minimal if and only if $H=0$.

\section{Main Results}

In this part, we define the type- 2 Smarandache ruled surfaces within Euclidean 3-space $\mathbf{E}^{3}$ referring to the frame $\left\{\mu_{1}, \mu_{2}, B\right\}$. Furthermore, we evaluate the sufficient and necessary conditions that enable these surfaces to be developable and minimal.

\section{1. $\mu_{1} \mu_{2}$ Type-2 Smarandache Ruled Surface}

Definition 5. For a regular curve $\psi=\psi(\sigma)$ in $\mathbf{E}^{3}$ related to the frame $\left\{\mu_{1}, \mu_{2}, B\right\}$, the $\mu_{1} \mu_{2}$ type-2 Smarandache ruled surface is given as

$$
\Omega=\Omega(\sigma, v)=\frac{1}{\sqrt{2}}\left(\mu_{1}(\sigma)+\mu_{2}(\sigma)\right)+v B(\sigma) .
$$

Theorem 6. Let $\Omega=\Omega(\sigma, v)$ be the $\mu_{1} \mu_{2}$ type-2 Smarandache ruled surface in $\mathbf{E}^{3}$ defined by (13). Then, we have

(1) $\Omega$ is a developable surface with asymptotic base curve $\psi(\sigma)$

(2) $\Omega$ is a minimal surface if and only if the type-2 Bishop curvatures satisfy the following equation

$$
k_{1}=k_{2} e^{\sigma+c}
$$

where $c$ is real constant.

Proof. Considering that the $\mu_{1} \mu_{2}$ type- 2 Smarandache ruled surface given by (13), then, the velocity vectors of $\Omega$ are given as follows:

$$
\begin{aligned}
& \Omega_{\sigma}=v k_{1} \mu_{1}+v k_{2} \mu_{2}-\left(\frac{k_{1}+k_{2}}{\sqrt{2}}\right) B, \\
& \Omega_{v}=B .
\end{aligned}
$$


From equation (15), we can obtain that the $\Omega$ 's quantities of fundamental forms are

$$
\begin{gathered}
E=v^{2} \tau^{2}+\frac{1}{2}\left(k_{1}+k_{2}\right)^{2}, \\
F=\frac{-1}{\sqrt{2}}\left(k_{1}+k_{2}\right), \\
G=1, \\
\ell=\left(\frac{k_{2}}{\tau}\right)\left[v k_{1}^{\prime}-\frac{k_{1}\left(k_{1}+k_{2}\right)}{\sqrt{2}}\right]-\left(\frac{k_{1}}{\tau}\right)\left[v k_{2}^{\prime}-\frac{k_{1}\left(k_{1}+k_{2}\right)}{\sqrt{2}}\right], \\
m=0, \\
n=0 .
\end{gathered}
$$

Consequently, from the above data, we obtain $K_{\Omega}$ and $H_{\Omega}$ of the $\mu_{1} \mu_{2}$ type- 2 Smarandache ruled surface given as follows:

$$
\begin{aligned}
& K_{\Omega}=0, \\
& H_{\Omega}=\frac{k_{1}^{\prime} k_{2}-k_{1} k_{2}^{\prime}}{2 v \tau^{3}} .
\end{aligned}
$$

Also, we use (12) to get the normal curvature, the geodesic curvature, and the geodesic torsion that associate $\psi(\sigma)$ on $\Omega$ as the following:

$$
\begin{aligned}
& \kappa_{n}=0, \\
& \kappa_{g}=-\frac{k_{1}^{2}}{\tau}, \\
& \tau_{g}=\frac{1}{\tau^{3}}\left[\tau^{2}\left(\frac{k_{1} k_{2}}{\tau}\right)^{\prime}-k_{1} k_{2} \tau^{\prime}\right] .
\end{aligned}
$$

So, the proof ended.

\section{2. $\mu_{1} B$ Type-2 Smarandache Ruled Surface}

Definition 7. For a regular curve $\psi=\psi(\sigma)$ in $\mathbf{E}^{3}$ related to the frame $\left\{\mu_{1}, \mu_{2}, B\right\}$, the $\mu_{1} B$ type- 2 Smarandache ruled surface is given as

$$
\Phi=\Phi(\sigma, v)=\frac{1}{\sqrt{2}}\left(\mu_{1}(\sigma)+B(\sigma)\right)+v \mu_{2}(\sigma)
$$

Theorem 8. Let $\Phi=\Phi(\sigma, v)$ be the $\mu_{1} B$ type-2 Smarandache ruled surface in $\mathbf{E}^{3}$ defined by (19). Then, we have

(1) If $k_{1} k_{2}=0$, then, $\Phi$ is a developable surface with the geodesic base curve

(2) $\Phi$ is a minimal surface with the geodesic base curve if and only if the type-2 Bishop curvatures satisfy the following differential equation

$$
\begin{gathered}
\left(k_{1}+\sqrt{2} v k_{2}\right)\left[k_{1}^{\prime}-k_{1}\left(k_{1}+\sqrt{2} v k_{2}\right)\right] \\
-k_{1}\left(\tau^{2}+k_{1}^{\prime}+\sqrt{2} v k_{2}^{\prime}\right)-2 k_{1} k_{2}^{2}=0 .
\end{gathered}
$$

Proof. Considering the $\mu_{1} B$ type- 2 Smarandache ruled surface given by (19), then, the velocity vectors of $\Phi$ are given as follows:

$$
\begin{aligned}
& \Phi_{\sigma}=\left(\frac{k_{1}}{\sqrt{2}}\right) \mu_{1}+\left(\frac{k_{2}}{\sqrt{2}}\right) \mu_{2}-\left(\frac{k_{1}+\sqrt{2} v k_{2}}{\sqrt{2}}\right) B, \\
& \Phi_{v}=\mu_{2} .
\end{aligned}
$$

From equation (21), the $\Phi$ 's quantities of fundamental forms are

$$
\begin{aligned}
& E=\frac{1}{2}\left[\tau^{2}+\left(k_{1}+\sqrt{2} v k_{2}\right)^{2}\right], \\
& F=\frac{k_{2}}{\sqrt{2}}, \\
& G=1, \\
& \ell=\frac{\left(k_{1}+\sqrt{2} v k_{2}\right)\left[k_{1}^{\prime}-k_{1}\left(k_{1}+\sqrt{2} v k_{2}\right)\right]-k_{1}\left[\tau^{2}+k_{1}^{\prime}+\sqrt{2} v k_{2}^{\prime}\right]}{\sqrt{2} \sqrt{k_{1}^{2}+\left(k_{1}+\sqrt{2} v k_{2}\right)^{2}}}, \\
& m=-\frac{k_{1} k_{2}}{\sqrt{k_{1}^{2}+\left(k_{1}+\sqrt{2} v k_{2}\right)^{2}}}, \\
& n=0 .
\end{aligned}
$$

Then, $K_{\Phi}$ and $H_{\Phi}$ of the $\mu_{1} B$ type-2 Smarandache ruled surface is given as follows:

$$
\begin{aligned}
& K_{\Phi}=-\frac{2 k_{1}^{2} k_{2}^{2}}{\left[k_{1}^{2}+\left(k_{1}+\sqrt{2} v k_{2}\right)^{2}\right]\left[\tau^{2}-k_{2}^{2}+\left(k_{1}+\sqrt{2} v k_{2}\right)^{2}\right]}, \\
& H_{\Phi}=\frac{\left(k_{1}+\sqrt{2} v k_{2}\right)\left[k_{1}^{\prime}-k_{1}\left(k_{1}+\sqrt{2} v k_{2}\right)\right]-k_{1}\left[\tau^{2}+k_{1}^{\prime}+\sqrt{2} v k_{2}^{\prime}\right]+2 k_{1} k_{2}^{2}}{\sqrt{2} \sqrt{k_{1}^{2}+\left(k_{1}+\sqrt{2} v k_{2}\right)^{2}}} .
\end{aligned}
$$


Furthermore, from (12), we have

$$
\begin{aligned}
& \kappa_{n}=\frac{\left(k_{1}^{\prime}-k_{1}^{2}\right)\left(k_{1}+\sqrt{2} v k_{2}\right)-k_{1}\left(\tau^{2}+k_{1}^{\prime}\right)}{\sqrt{2} \sqrt{k_{1}^{2}+\left(k_{1}+\sqrt{2} v k_{2}\right)^{2}}}, \\
& \kappa_{g}=0, \\
& \tau_{g}=-\frac{k_{1}^{2} k_{2}\left(k_{1}+\sqrt{2} v k_{2}\right)}{k_{1}^{2}+\left(k_{1}+\sqrt{2} v k_{2}\right)^{2}},
\end{aligned}
$$

which replies to the above theorem.

\section{3. $\mu_{2} B$ Type-2 Smarandache Ruled Surface}

Definition 9. For a regular curve $\psi=\psi(\sigma)$ in $\mathbf{E}^{3}$ related to the frame $\left\{\mu_{1}, \mu_{2}, B\right\}$, the $\mu_{2} B$ type- 2 Smarandache ruled surface is given as

$$
\Psi=\Psi(\sigma, v)=\frac{1}{\sqrt{2}}\left(\mu_{2}(\sigma)+B(\sigma)\right)+v \mu_{1}(\sigma) .
$$

Theorem 10. Let $\Psi=\Psi(\sigma, v)$ be the $\mu_{2} B$ type-2 Smarandache ruled surface in $\mathbf{E}^{3}$ defined by (25). Then, we have

(1) If $k_{1} k_{2}=0$, then, $\Psi$ is a developable surface with the principal base curve

(2) $\Psi$ is a minimal surface if and only if the type-2 Bishop curvatures satisfy the following differential equation

$$
\begin{gathered}
k_{2}\left(\tau^{2}+k_{2}^{\prime}+\sqrt{2} v k_{1}^{\prime}\right)-2 k_{1}^{2} k_{2}-\left(k_{2}+\sqrt{2} v K_{1}\right) \\
\cdot\left[k_{2}^{\prime}-k_{2}\left(k_{2}+\sqrt{2} v k_{1}\right)\right]=0 .
\end{gathered}
$$

Proof. Considering the $\mu_{2} B$ type-2 Smarandache ruled surface given by (25), then, the velocity vectors of $\Psi$ are given as follows:

$$
\begin{gathered}
\Psi_{\sigma}=\left(\frac{k_{1}}{\sqrt{2}}\right) \mu_{1}+\left(\frac{k_{2}}{\sqrt{2}}\right) \mu_{2}-\left(\frac{k_{2}+\sqrt{2} v k_{2}}{\sqrt{2}}\right) B, \\
\Psi_{v}=\mu_{1} .
\end{gathered}
$$

From equation (27), the $\Psi$ 's quantities of fundamental forms are

$$
\begin{aligned}
& E=\frac{1}{2}\left[\tau^{2}+\left(k_{2}+\sqrt{2} v k_{1}\right)^{2}\right], \\
& F=\frac{k_{1}}{\sqrt{2}}, \\
& G=1, \\
& \ell=\frac{k_{2}\left[\tau^{2}+k_{2}^{\prime}+\sqrt{2} v k_{1}^{\prime}\right]-\left(k_{2}+\sqrt{2} v k_{1}\right)\left[k_{2}^{\prime}-k_{2}\left(k_{2}+\sqrt{2} v k_{1}\right)\right]}{\sqrt{2} \sqrt{k_{2}^{2}+\left(k_{2}+\sqrt{2} v k_{1}\right)^{2}}}, \\
& m=\frac{k_{1} k_{2}}{\sqrt{k_{2}^{2}+\left(k_{2}+\sqrt{2} v k_{1}\right)^{2}}}, \\
& n=0 .
\end{aligned}
$$

The $K_{\Psi}$ and $H_{\Psi}$ of the $\mu_{2} B$ type-2 Smarandache ruled surface given as follows:

$$
\begin{aligned}
& K_{\Psi}=-\frac{2 k_{1}^{2} k_{2}^{2}}{\left[k_{2}^{2}+\left(k_{2}+\sqrt{2} v k_{1}\right)^{2}\right]\left[\tau^{2}-k_{1}^{2}+\left(k_{2}+\sqrt{2} v k_{1}\right)^{2}\right]}, \\
& H_{\Psi}=\frac{k_{2}\left(\tau^{2}+k_{2}^{\prime}+\sqrt{2} v k_{1}^{\prime}\right)-2 k_{1}^{2} k_{2}-\left(k_{2}+\sqrt{2} v k_{1}\right)\left[k_{2}^{\prime}-k_{2}\left(k_{2}+\sqrt{2} v k_{1}\right)\right]}{\sqrt{2} \sqrt{k_{2}^{2}+\left(k_{2}+\sqrt{2} v k_{1}\right)^{2}}} .
\end{aligned}
$$

So, the proof ended.

Also, from (12), we have

$$
\begin{array}{lc} 
& \kappa_{g}=\frac{k_{1}\left(k_{2}+\sqrt{2} v k_{1}\right)}{\sqrt{k_{2}^{2}+\left(k_{2}+\sqrt{2} v k_{1}\right)^{2}}}, \\
\kappa_{n}=\frac{k_{2}\left(\tau^{2}+k_{2}^{\prime}\right)-\left(k_{2}^{\prime}-k_{1}^{2}\right)\left(k_{2}+\sqrt{2} v k_{1}\right)}{\sqrt{2} \sqrt{k_{2}^{2}+\left(k_{2}+\sqrt{2} v k_{1}\right)^{2}}}, & \tau_{g}=\frac{k_{1}^{2} k_{2}\left(k_{2}+\sqrt{2} v k_{1}\right)}{k_{2}^{2}+\left(k_{2}+\sqrt{2} v k_{1}\right)^{2}} .
\end{array}
$$




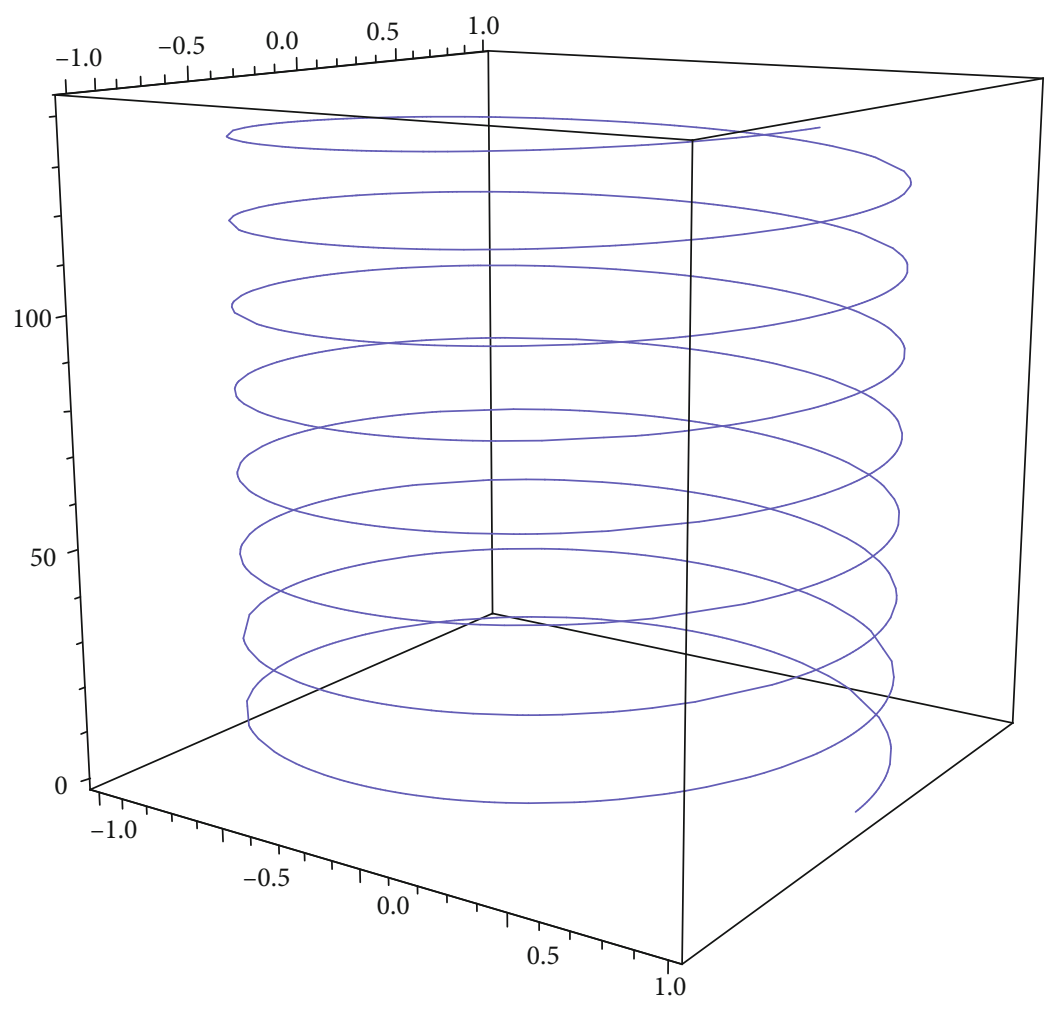

FIgURE 1: Curve $\psi=\psi(\sigma)$.

Then, equations (29) and (30) complete the proof.

3.4. Example. Let $\psi$ be a circular helix parameterized as $\psi($ $\sigma)=(\cos (\sigma / 3), \sin (\sigma / 3),(2 \sqrt{2} \sigma / 3))$ (see Figure 1). Then, we have

$$
\begin{aligned}
& T(\sigma)=\left(-\frac{1}{3} \sin \left(\frac{\sigma}{3}\right), \frac{1}{3} \cos \left(\frac{\sigma}{3}\right), \frac{2 \sqrt{2}}{3}\right), \\
& N(\sigma)=\left(-\cos \left(\frac{\sigma}{3}\right),-\sin \left(\frac{\sigma}{3}\right), 0\right), \\
& B(\sigma)=\left(\frac{2 \sqrt{2}}{3} \sin \left(\frac{\sigma}{3}\right),-\frac{2 \sqrt{2}}{3} \cos \left(\frac{\sigma}{3}\right), \frac{1}{3}\right) .
\end{aligned}
$$

Then, $\tau=2 \sqrt{2} / 9 \neq 0$ and $\theta(\sigma)=\int_{0}^{\sigma}(1 / 9) d \sigma=\sigma / 9$. From (4), we get $\kappa_{1}(\sigma)=-(2 \sqrt{2} / 9) \cos (\sigma / 9), \kappa_{2}(\sigma)=-(2 \sqrt{2} / 9)$ $\sin (\sigma / 9)$. Also, we have

$$
\begin{aligned}
\mu_{1}(\sigma)= & \left(-\cos \left(\frac{\sigma}{9}\right) \cos \left(\frac{\sigma}{3}\right)-\frac{1}{3} \sin \left(\frac{\sigma}{9}\right)\right. \\
& \cdot \sin \left(\frac{\sigma}{3}\right), \frac{1}{3} \cos \left(\frac{\sigma}{3}\right) \sin \left(\frac{\sigma}{9}\right) \\
& \left.-\cos \left(\frac{\sigma}{9}\right) \sin \left(\frac{\sigma}{3}\right), \frac{2 \sqrt{2}}{9} \sin \left(\frac{\sigma}{9}\right)\right),
\end{aligned}
$$

$$
\begin{aligned}
\mu_{2}(\sigma)= & \left(-\cos \left(\frac{\sigma}{3}\right) \sin \left(\frac{\sigma}{9}\right)+\frac{1}{3} \cos \left(\frac{\sigma}{9}\right) \sin \left(\frac{\sigma}{3}\right),\right. \\
& -\frac{1}{3} \cos \left(\frac{\sigma}{9}\right) \cos \left(\frac{\sigma}{3}\right)-\sin \left(\frac{\sigma}{9}\right) \sin \left(\frac{\sigma}{3}\right), \\
& \left.-\frac{2 \sqrt{2}}{9} \cos \left(\frac{\sigma}{9}\right)\right)
\end{aligned}
$$

The $\mu_{1} \mu_{2}$ type- 2 Smarandache ruled surface $\Omega(\sigma, v)$ is (see Figure 2)

$$
\begin{aligned}
\Omega(\sigma, v)= & \left(\frac { 1 } { \sqrt { 2 } } \left\{\frac{1}{3} \sin \left(\frac{\sigma}{3}\right)\left(\cos \left(\frac{\sigma}{9}\right)-\sin \left(\frac{\sigma}{9}\right)\right)\right.\right. \\
& \left.-\cos \left(\frac{\sigma}{3}\right)\left(\sin \left(\frac{\sigma}{9}\right)+\cos \left(\frac{\sigma}{9}\right)\right)\right\} \\
& +\frac{2}{3} v \sin \left(\frac{\sigma}{3}\right), \frac{1}{\sqrt{2}}\left\{\frac { 1 } { 3 } \operatorname { c o s } ( \frac { \sigma } { 3 } ) \left(\sin \left(\frac{\sigma}{9}\right)\right.\right. \\
& \left.-\cos \left(\frac{\sigma}{9}\right)\right)-\sin \left(\frac{\sigma}{3}\right)\left(\cos \left(\frac{\sigma}{9}\right)\right. \\
& \left.\left.+\sin \left(\frac{\sigma}{9}\right)\right)\right\}-\frac{2}{3} v \cos \left(\frac{\sigma}{3}\right), \frac{2}{3} \\
& \left.\cdot\left(\sin \left(\frac{\sigma}{9}\right)-\cos \left(\frac{\sigma}{9}\right)+\frac{v}{3}\right)\right) .
\end{aligned}
$$




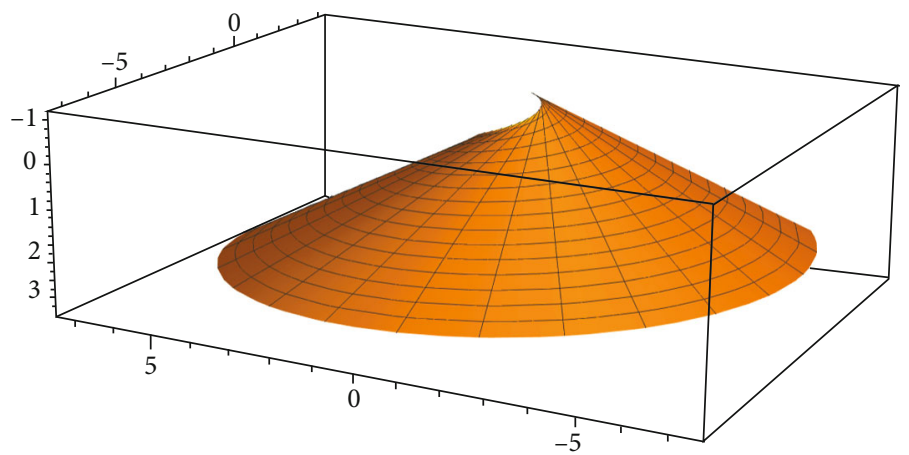

Figure 2: $\mu_{1} \mu_{2}$ type-2 Smarandache ruled surface $\Omega(\sigma, v)$.

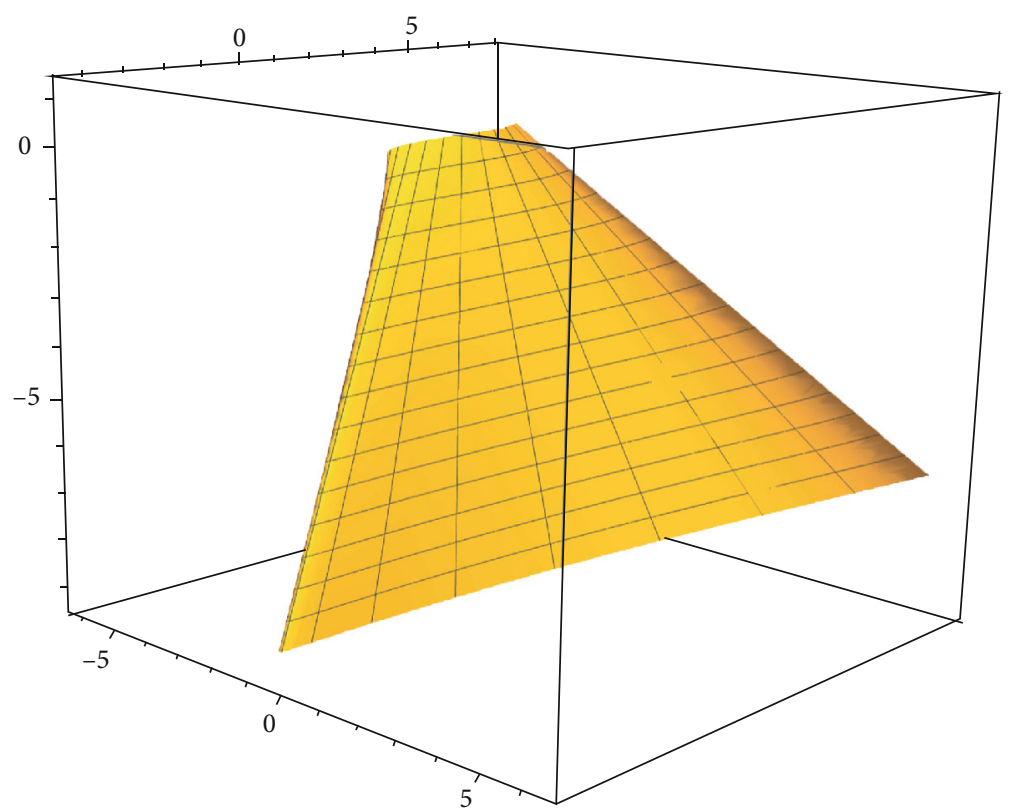

Figure 3: $\mu_{1} B$ type-2 Smarandache ruled surface $\Phi(\sigma, v)$.

The $\mu_{1} B$ type-2 Smarandache ruled surface $\Phi(\sigma, v)$ is (see Figure 3)

$$
\begin{aligned}
\Phi(\sigma, v)= & \left(\frac{1}{3} \sin \left(\frac{\sigma}{3}\right)\left(v \cos \left(\frac{\sigma}{9}\right)-\frac{1}{\sqrt{2}} \sin \left(\frac{\sigma}{9}\right)\right)\right. \\
& -\cos \left(\frac{\sigma}{3}\right)\left(v \sin \left(\frac{\sigma}{9}\right)+\frac{1}{\sqrt{2}} \cos \left(\frac{\sigma}{9}\right)\right) \\
& +\frac{2}{3} \sin \left(\frac{\sigma}{3}\right), \frac{1}{3} \cos \left(\frac{\sigma}{3}\right)\left(\frac{1}{\sqrt{2}} \sin \left(\frac{\sigma}{3}\right)\right. \\
& \left.-v \cos \left(\frac{\sigma}{3}\right)\right)-\sin \left(\frac{\sigma}{3}\right)\left(v \sin \left(\frac{\sigma}{3}\right)\right. \\
& \left.+\frac{1}{\sqrt{2}} \cos \left(\frac{\sigma}{3}\right)\right)-\frac{2}{3} \cos \left(\frac{\sigma}{3}\right), \frac{2}{3} \\
& \left.+\left(\sin \left(\frac{\sigma}{9}\right)-\sqrt{2} v \cos \left(\frac{\sigma}{9}\right)+\frac{1}{2 \sqrt{2}}\right)\right)
\end{aligned}
$$

The $\mu_{2} B$ type- 2 Smarandache ruled surface $\Psi(\sigma, v)$ is (see Figure 4)

$$
\begin{aligned}
\Psi(\sigma, v)= & \left(\frac{1}{3} \sin \left(\frac{\sigma}{3}\right)\left(\frac{1}{\sqrt{2}} \cos \left(\frac{\sigma}{9}\right)-v \sin \left(\frac{\sigma}{9}\right)\right)\right. \\
& -\cos \left(\frac{\sigma}{3}\right)\left(\frac{1}{\sqrt{2}} \sin \left(\frac{\sigma}{9}\right)+v \cos \left(\frac{\sigma}{9}\right)\right) \\
& +\frac{2}{3} \sin \left(\frac{\sigma}{3}\right), \frac{1}{3} \cos \left(\frac{\sigma}{3}\right)\left(v \sin \left(\frac{\sigma}{3}\right)\right. \\
& \left.-\frac{1}{\sqrt{2}} \cos \left(\frac{\sigma}{3}\right)\right)-\sin \left(\frac{\sigma}{3}\right)\left(\frac{1}{\sqrt{2}} \sin \left(\frac{\sigma}{3}\right)\right. \\
& \left.+v \cos \left(\frac{\sigma}{3}\right)\right)-\frac{2}{3} \cos \left(\frac{\sigma}{3}\right), \frac{2}{3} \\
& \left.\cdot\left(\sqrt{2} v \sin \left(\frac{\sigma}{9}\right)-\cos \left(\frac{\sigma}{9}\right)+\frac{1}{2 \sqrt{2}}\right)\right)
\end{aligned}
$$




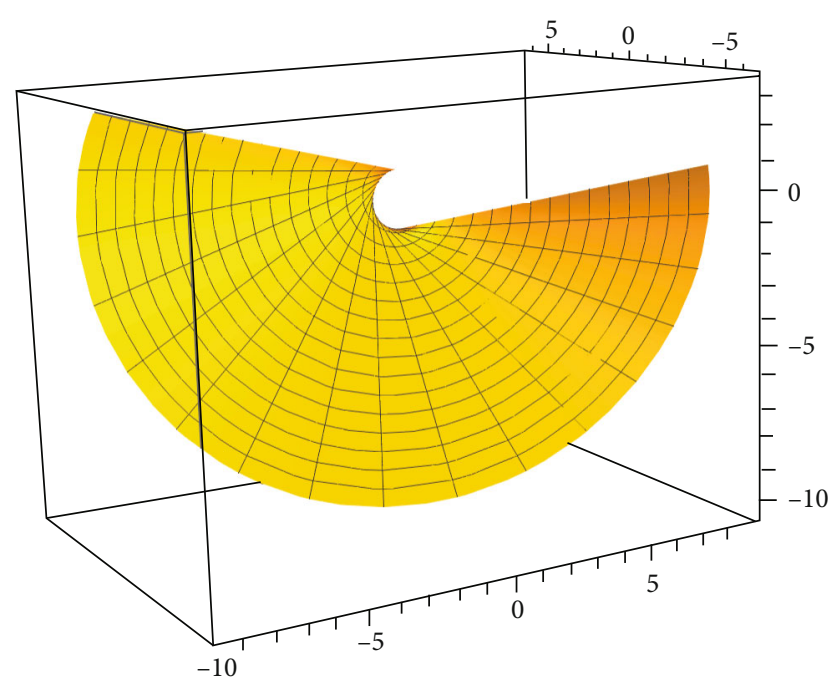

Figure 4: $\mu_{2} B$ type-2 Smarandache ruled surface $\Psi(\sigma, v)$.

\section{Conclusion}

The study of ruled surfaces with different moving frames is one of the most interesting points of this paper. The researchers found that these surfaces could be developed in a minimal amount of time. In this work, we describe and study type- 2 Smarandache ruled surfaces, which are a specific form of ruled surfaces. We create the essential and adequate circumstances for these surfaces to be developable in a minimal amount of time.

\section{Data Availability}

No data is used in this study.

\section{Conflicts of Interest}

The authors declare no competing interest.

\section{Authors' Contributions}

All authors have equal contributions and finalized the study.

\section{Acknowledgments}

The authors extend their appreciation to the Deanship of Scientific Research at Imam Mohammad Ibn Saud Islamic University for funding this work through Research Group no. RG-21-09-04.

\section{References}

[1] M. P. Do Carmo, Differential Geometry of Curves and Surfaces: Revised and Updated Second Edition, Courier Dover Publications, 2016.

[2] D. J. Struik, Lectures on Classical Differential Geometry, AddisonWesley, Dover, 2nd edition edition, 1988.

[3] G. Hu, H. Cao, J. Wu, and G. Wei, "Construction of developable surfaces using generalized C-Bézier bases with shape parameters," Computational and Applied Mathematics, vol. 39, no. 3, 2020 .
[4] W. Y. Lam, "Minimal surfaces from infinitesimal deformations of circle packings," Advances in Mathematics, vol. 362, article 106939, 2020.

[5] S. Ouarab, A. O. Chahdi, and M. Izid, "Ruled surfaces with alternative moving frame in Euclidean 3-space," International Journal of Mathematical Sciences and Engineering Applications, vol. 12, no. 2, pp. 43-58, 2018.

[6] S. Ouarab, A. O. Chahdi, and M. Izid, "Ruled surface generated by a curve lying on a regular surface and its characterizations," Journal for Geometry and Graphics, vol. 24, no. 2, pp. 257-267, 2020.

[7] S. Ouarab, "Smarandache ruled surfaces according to FrenetSerret frame of a regular curve in $E^{3}$," Abstract and Applied Analysis, vol. 2021, 8 pages, 2021.

[8] C. Ashbacher, "Smarandache geometries," The American Mathematical Monthly, vol. 8, no. 1-3, pp. 212-215, 1997.

[9] L. R. Bishop, "There is more than one way to frame a curve," The American Mathematical Monthly, vol. 82, no. 3, pp. 246-251, 1975.

[10] H. Iseri, Smarandache Manifolds, American Res, Press, Mansfield University, PA, 2002.

[11] L. Mao, Smarandache Geometries \& Map Theory with Applications, Chinese Branch Xiquan House, Academy of Mathematics and Systems Chinese Academy of Sciences, Beijing, China, 2006.

[12] E. M. Solouma and M. M. Wageeda, "Special Smarandache curves according to Bishop frame in Euclidean space-time," International Journal of Mathematical Combinatorics, vol. 1, pp. 1-9, 2017.

[13] E. M. Solouma, "Special SMRANDACHE curves recording by curves on a spacelike surface in Minkowski space-time," Ponte, vol. 73, no. 2, pp. 251-263, 2017.

[14] E. M. Solouma, "Special equiform Smarandache curves in Minkowski space-time," Journal of the Egyptian Mathematical Society, vol. 25, no. 3, pp. 319-325, 2017.

[15] E. M. Solouma, "Type-2 spacelike Bishop frame and an application to spherical image in Minkowski space-time," International Journal of Applied and Computational Mathematics, vol. 3, no. 4, pp. 3575-3591, 2017.

[16] E. M. Solouma and W. M. Mahmoud, "On spacelike equiform Bishop Smarandache curves on $S_{1}^{2}$," Journal of the Egyptian Mathematical Society, vol. 27, no. 1, pp. 1-17, 2019.

[17] E. M. Solouma, "Equiform spacelike Smarandache curves of anti-Eqiform Salkowski curve according to Equiform frame," International Journal of Mathematical Analysis, vol. 15, no. 1, pp. 43-59, 2021.

[18] M. Turgut and S. Ylmaz, "Smarandache curves in Minkowski space-time," International Journal of Mathematical Combinatorics, vol. 3, pp. 51-55, 2008.

[19] K. Tasköprü and M. Tosun, "Smarandache curves on $S^{2}$," Boletim da Sociedade Paraneanse de Matematica, vol. 32, no. 1, pp. 51-59, 2014.

[20] M. Çetin, Y. Tunçer, and M. K. Karacan, "Smarandache curves according to Bishop frame in Euclidean 3- space," General Mathematics Notes, vol. 20, no. 2, pp. 50-66, 2014.

[21] S. Yilmaz and M. Turgut, "A new version of Bishop frame and an application to spherical images," Journal of Mathematical Analysis and Applications, vol. 371, no. 2, pp. 764-776, 2010.

[22] J. L. M. Barbosa and A. G. Colares, Minimal Surface in $R^{3}$, Springer Verlag, Berlin Heidelberg, 1986.

[23] B. O’Neill, Semi-Riemannian Geometry with Applications to Relativity, Academic press, New York, 1983. 